\title{
ALGUNOS CRITERIOS BIOLÓGICOS SOBRE EL PROCESO DE EUTROFIZACIÓN A ORILLAS DE SEIS LOCALIDADES DEL LAGO TITIKAKA
}

\author{
Francisco Fontúrbel Rada ${ }^{1}$
}

\section{Resumen}

Se evaluaron las condiciones ambientales de 6 sitios a orillas del lago Titikaka (La Paz, Bolivia), encontrándose diferentes grados de eutrofización. Se emplearon 4 indicadores de evaluación rápida: macrófitas flotantes y sumergidas, fitoplancton y propiedades organolépticas, complementándolos con análisis de $\mathrm{pH}$ y micro-meso fauna. Se encontraron diferentes grados de afectación por el proceso eutrófico en parte debido a la influencia humana y la contaminación de los sitios de estudio. Se analizó la relevancia de cada indicador en este caso particular.

Palabras clave: Lago Titikaka, eutrofización, indicadores biológicos, fitoplancton.

\begin{abstract}
Environmental conditions were evaluated for 6 study sites at Titikaka's lake margins (La Paz, Bolivia), where I founded 4 different degrees of eutrophic processes. 4 fast-evaluation indicator parameters were used: floating and submerged macrophytes, phytoplankton and organoleptic properties, which were complemented with $\mathrm{pH}$ and micro-middle fauna analysis. Different affectations degrees of eutrophic process were founded in associated in part to human influence and contamination at the study sites. Indicator relevancy was analyzed for each particular case.
\end{abstract}

Key words: Titikaka Lake, eutrophication, biologic indicators, phytoplankton.

\section{Introducción}

En la actualidad, la eutrofización cultural (asociada a la hipertrofia) es uno de los problemas con mayor repercusión sobre los cuerpos de agua y el medio ambiente (Dolbeth et al., 2003). La constante descarga de desechos sólidos y líquidos en lagos y lagunas ha ocasionado el desarrollo de este complejo problema en cuerpos de agua de todo el mundo, y el Lago Titikaka no es la excepción.

La eutrofización es un fenómeno complejo que involucra factores climáticos, físicos y biológicos (Dolbeth et al., 2003; Howarth et al., 2000), ocasionando cambios en la diversidad y la abundancia de las especies (Agatz et al., 1999; Barrón et al., 2003; Dolbeth et al., 2003; Lang , 1997; Weithoff et al., 2000). Si bien las implicancias de este proceso son muy complejas (Kim et al., 2001) -incluso a nivel de metabolismo (Macek et al., 2000)- algunos factores indicadores simples (Schroll, 2002) pueden ser usados para una detección y evaluación rápida del proceso de eutrofización.

Esta trabajo hace referencia a un hecho no esperado durante un proyecto de investigación realizado en el Lago Titikaka, donde se documentó la presencia de indicios de eutrofización en los seis sitios estudiados (Fontúrbel et al., 2003), y por lo tanto pretende dar un informe preliminar de la situación observada.

\section{Materiales y métodos \\ Sitios de muestreo}

Se tomaron 6 sitios de muestreo (Tabla 1 y Figura 1) a orillas de la parte boliviana del Lago Titikaka, escogidos de acuerdo a su relevancia y aspectos paisajísticos.

Tabla 1: Identificación y localización de los sitios de estudio

\begin{tabular}{lll}
\hline \hline Sitio & Localidad & Coordenadas \\
\hline \hline 1 & Ajaría Grande & $16^{\circ} 1^{\prime} 58,71^{\prime \prime S} 68^{\circ} 45^{\prime} 43,87^{\prime \prime} \mathrm{W}$ \\
\hline 2 & Toke-Pucuru & $16^{\circ} 1^{\prime} 16,81^{\prime \prime S} 68^{\circ} 49^{\prime} 25,84^{\prime \prime} \mathrm{W}$ \\
\hline 3 & Uricachi & $16^{\circ} 3^{\prime} 40,18^{\prime \prime} \mathrm{S} 68^{\circ} 50^{\prime} 16,84^{\prime \prime} \mathrm{W}$ \\
\hline 4 & Huatajata & $16^{\circ} 12^{\prime} 35.58^{\prime \prime} \mathrm{S} 68^{\circ} 41^{\prime} 11,54^{\prime \prime} \mathrm{W}$ \\
\hline 5 & Sorejapa & $16^{\circ} 12^{\prime} 29,14^{\prime \prime} \mathrm{S} 68^{\circ} 38^{\prime} 28,12^{\prime \prime} \mathrm{W}$ \\
\hline 6 & Huarina & $16^{\circ} 13^{\prime} 26,58^{\prime \prime} \mathrm{S} 68^{\circ} 35^{\prime} 9,66^{\prime \prime} \mathrm{W}$ \\
\hline
\end{tabular}

\section{Análisis de parámetros}

En base a referencias de Pérez $(1998,2002)$ se analizaron los siguientes parámetros: $\mathrm{pH}$ del agua, abundancia y diversidad de fitoplancton, vegetación sumergida, vegetación flotante, micro y meso fauna, y adicionalmente se registró el grado de contaminación. Para todos los casos se tomaron muestras por triplicado, las que fueron analizadas en los

\footnotetext{
${ }^{1}$ Unidad de Post-Grado Universidad Loyola. Maestría en Ingeniería de Medio Ambiente y Recursos Naturales. Av. Busch \# 1191, La Paz, Bolivia.

Autor: Casilla postal \# 180, La Paz, Bolivia. Av. 20 de Octubre \# 2005, Dep. 301, La Paz, Bolivia. Correo electrónico: fonturbel $@$,mbotanica.zzn.com
} 


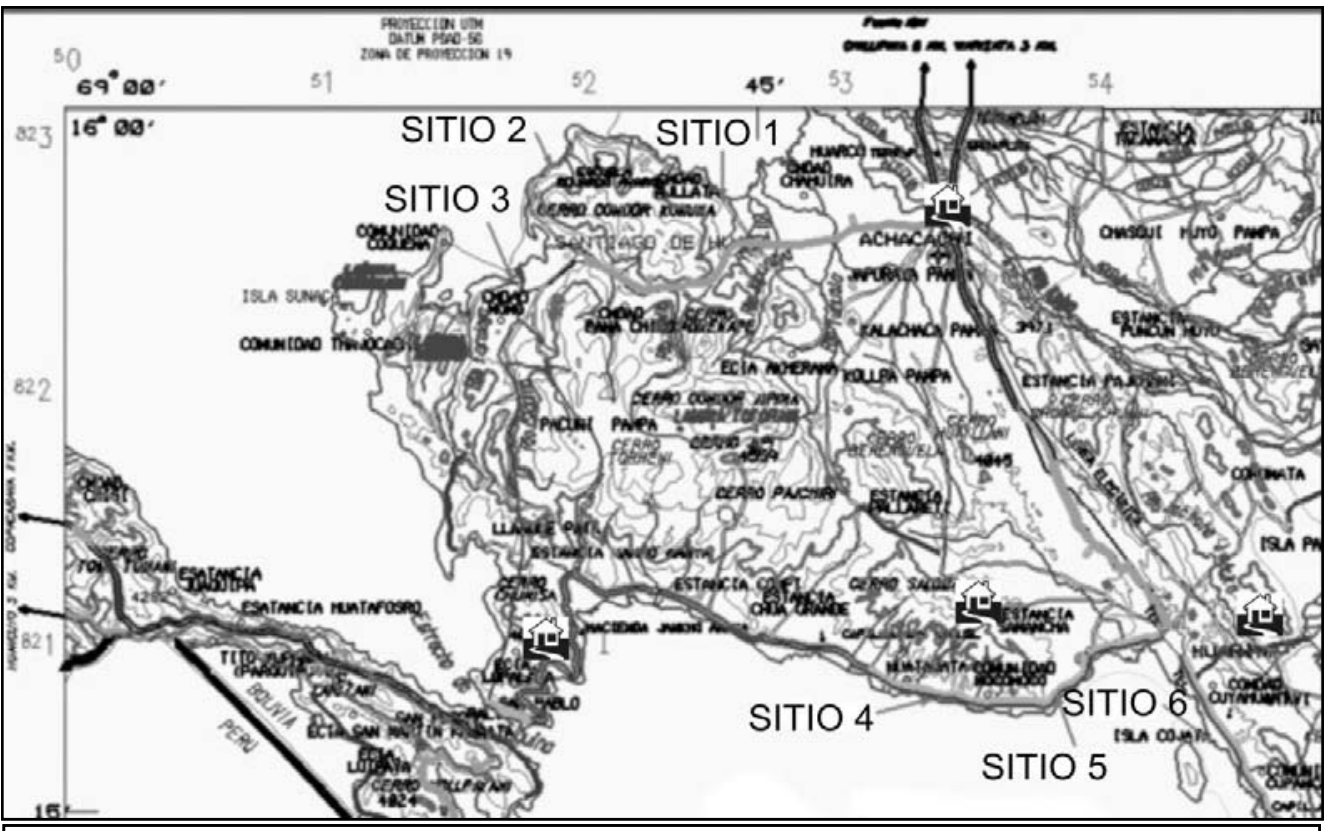

Fig. 1: Mapa de ubicación de los sitios de estudio. Los sitios más poblados están marcados con el ícono en forma de casa.

laboratorios de Calidad Ambiental, Suelos y Limnología del Instituto de Ecología de la Universidad Mayor de San Andrés.

\section{Indicadores de eutrofización}

Se tomaron como indicadores de eutrofización: (1) la presencia y cobertura de las macrófitas flotantes Lemna spp. y Azolla spp., (2) la diversidad y cobertura de la vegetación sumergida, (3) la cantidad y diversidad de fitoplancton, y (4) las propiedades organolépticas del agua (turbidez y olor).

\section{Resultados}

Los 6 sitios estudiados presentaban algún tipo de contaminación (Tabla 2), los diferentes parámetros indicadores empleados mostraron diferentes grados de eutrofización (Tabla 3). El grado estimado de eutrofización para cada sitio también se muestra en la Tabla 3.

Tabla 2: Descripción breve de la contaminación encontrada en cada uno de los sitios de estudio.

\begin{tabular}{ll}
\hline \hline Sitio & Contaminación \\
\hline \hline 1 & Heces animales y residuos sólidos \\
\hline 2 & Presencia de algunos restos plásticos en las orillas \\
\hline 3 & $\begin{array}{l}\text { Fuerte contaminación por desechos humanos, } \\
\text { residuos sólidos y líquidos }\end{array}$ \\
\hline 4 & $\begin{array}{l}\text { Contaminación extrema por todo tipo de residuos y } \\
\text { desechos }\end{array}$ \\
\hline 5 & Escasa contaminación por residuos sólidos \\
\hline 6 & $\begin{array}{l}\text { Contaminación intermedia localizada, presencia de } \\
\text { residuos sólidos }\end{array}$ \\
\hline
\end{tabular}

\section{Discusión}

Si bien los 6 sitios de estudio registrados presentan algún grado de contaminación, la manifestación de los procesos eutróficos no está directamente relacionada con la contaminación ni con la cantidad de habitantes de las poblaciones cercanas ya que, por la extensión del lago, los procesos eutróficos todavía se va dando de manera localizada, variando de un sitio a otro de acuerdo con la capacidad de resilencia, asociada la presencia de algunas macrófitas, principalmente Schoenoplectus californicus ssp. totora.

Para esta evaluación preliminar se emplearon indicadores sencillos de evaluación situacional rápida como sugiere Schroll (2002), en base a ciertos criterios biológicos, que para este caso son: (1) presencia y cobertura de macrófitas flotantes, especialmente Lemna spp. y Azolla spp., características de los procesos eutróficos (Rodríguez, 2002), normalmente invasoras (RAMSAR, 1999) que se desarrollan por el incremento de carbono orgánico, nitrógeno y fósforo provenientes de los desechos orgánicos (Ferreira, 2001); (2) presencia de macrófitas sumergidas (Barrón et al., 2003; Dolbeth et al., 2003; McCook, 1999) que se reducen en abundancia y diversidad (García, 2003) por la falta de luz y oxígeno (Weisner et al., 1997), constituyéndose en un indicador relativamente bueno del avance del proceso eutrófico a mediano plazo (RAMSAR, 1999; Rodríguez, 2002); (3) abundancia y diversidad de fitoplancton, ya que los procesos eutróficos suelen producir un aumento de los productores primarios (Agatz et al., 1999; Dolbeth et al., 2003; Weisner et al., 1997; Weithoff et al., 2000), pero con una considerable reducción de la diversidad por la desaparición de los organismos estenotolerantes a ciertos nutrientes (Agatz et al., 1999, Kim et al., 2001; Weithoff et al., 2000), ocasionando una reducción de 
Tabla 3: Resultados de los criterios de biológicos empleados para los sitios de estudio y ranking de eutrofización.

\begin{tabular}{|c|c|c|c|c|c|c|c|}
\hline Sitio & $\begin{array}{l}\text { Macrófitas } \\
\text { flotantes } \\
\end{array}$ & $\begin{array}{l}\text { Vegetación } \\
\text { sumergida } \\
\end{array}$ & Fitoplancton & $\begin{array}{l}\text { Propiedades } \\
\text { organolépticas } \\
\end{array}$ & $\begin{array}{l}\mathrm{pH} \\
\text { agua } \\
\end{array}$ & Fauna & $\begin{array}{l}\text { Ranking de } \\
\text { eutrofización }\end{array}$ \\
\hline 1 & $\begin{array}{l}\text { Poca cantidad } \\
\text { de Azolla } \mathrm{sp} . \mathrm{y} \\
\text { Lemna sp. } \\
(\sim 10 \%)\end{array}$ & $\begin{array}{l}\text { Abundante } \\
\text { Myriophyllum } \\
\text { quitense, otras muy } \\
\text { escasas }\end{array}$ & $\begin{array}{l}\text { Abundante, poco } \\
\text { diverso con } \\
\text { predominancia de } \\
\text { pirrófitas y } \\
\text { diatomeas }\end{array}$ & $\begin{array}{l}\text { Aguas } \\
\text { ligeramente } \\
\text { turbias, muy poco } \\
\text { olor }\end{array}$ & 7,85 & $\begin{array}{l}\text { Moluscos } \\
\text { (Planorbidae) y } \\
\text { anélidos } \\
\text { (Tubificidae) }\end{array}$ & Ligera \\
\hline 2 & Ausentes & $\begin{array}{l}\text { Abundante, } 10 \% \text { S. } \\
\text { californicus ssp. } \\
\text { Totora, } 15 \% \text { Chara } \\
\text { sp., } 15 \% \text { Elodea sp., } \\
\text { presencia de Volvox } \\
\text { sp. }\end{array}$ & $\begin{array}{l}\text { Medianamente } \\
\text { abundante, bastante } \\
\text { diverso }\end{array}$ & $\begin{array}{l}\text { Aguas } \\
\text { ligeramente } \\
\text { turbias, } \\
\text { prácticamente sin } \\
\text { olor }\end{array}$ & 7,30 & $\begin{array}{l}\text { Alta diversidad de } \\
\text { moluscos, anélidos e } \\
\text { insectos. Larvas de } \\
\text { dípteros y } \\
\text { coleópteros. Anuros } \\
\text { pequeños }\end{array}$ & Ausente \\
\hline 3 & $\begin{array}{l}35 \% \text { de la } \\
\text { superficie } \\
\text { cubierta por } \\
\text { Lemna minor y } \\
\text { Azolla } \text { sp. }\end{array}$ & $\begin{array}{l}\text { Muy pocas } \\
\text { macrófitas de Chara } \\
\text { y clorófitas }\end{array}$ & $\begin{array}{l}\text { Medianamente } \\
\text { abundante, poco } \\
\text { diverso }\end{array}$ & $\begin{array}{l}\text { Aguas turbias de } \\
\text { muy mal olor }\end{array}$ & 7,23 & $\begin{array}{l}\text { Pocos moluscos } \\
\text { pequeños e insectos. } \\
\text { Presencia de } \\
\text { oligoquetos } \\
\text { (Tubificidae) }\end{array}$ & Avanzada \\
\hline 4 & $\begin{array}{l}\text { Presencia de } \\
\text { Lemna } \text { sp. } \\
(\sim 20 \%)\end{array}$ & $\begin{array}{l}5 \% \text { de } S . \text { californicus } \\
\text { ssp. totora, no hay } \\
\text { otra vegetación } \\
\text { sumergida }\end{array}$ & $\begin{array}{l}\text { Muy abundante } \\
\text { pero poco diverso, } \\
\text { predominancia de } \\
\text { diatomeas }\end{array}$ & $\begin{array}{l}\text { Aguas claras con } \\
\text { olor fétido }\end{array}$ & 6,76 & Ausente & Media \\
\hline 5 & $\begin{array}{l}\text { Poca cantidad } \\
\text { de Azolla } \text { sp. y } \\
\text { Lemna } \mathrm{sp} . \\
(<10 \%)\end{array}$ & $\begin{array}{l}\text { Abundante, } 10 \% \text { de } \\
\text { coebrtura de Chara } \\
\text { sp. y Potamogeton } \\
\text { pectinatus; musgos } \\
\text { sumergidos }\end{array}$ & $\begin{array}{l}\text { Escaso y poco } \\
\text { diverso } \\
\text { (predominan } \\
\text { diatomeas) }\end{array}$ & $\begin{array}{l}\text { Aguas claras y } \\
\text { limpias, casi sin } \\
\text { olor }\end{array}$ & 7,61 & $\begin{array}{l}\text { Pequeños arácnidos } \\
\text { y abundantes } \\
\text { oligoquetos } \\
\text { (Tubificidae) }\end{array}$ & Incipiente \\
\hline 6 & $\begin{array}{l}\text { Presencia de } \\
\text { Azolla } \mathrm{sp} . \\
(\sim 20 \%)\end{array}$ & $\begin{array}{l}\text { Macrófitas casi } \\
\text { ausentes, presencia } \\
\text { de unas pocas } \\
\text { clorófitas }\end{array}$ & Muy escaso & $\begin{array}{l}\text { Aguas } \\
\text { ligeramente } \\
\text { turbias, casi sin } \\
\text { olor }\end{array}$ & 7,69 & Ausente & Ligera \\
\hline
\end{tabular}

la diversidad de los demás niveles tróficos por medio de un aparente control bottom-up (Dolbeth et al., 2003; Koivula \& Rönkä, 1998), que puede a su vez ocasionar un fenómeno tipo top-down en otras redes tróficas (Manca et al., 2000), o darse una combinación de ellos (Achá \& Fontúrbel, 2003); (4) las propiedades organolépticas del agua -turbidez y olor- están indirectamente relacionadas con el proceso eutrófico, la turbidez puede deberse al incremento en la cantidad de fitoplancton por unidad de volumen (Kim et al., 2001; Schroll, 2002; Weisner et al., 1997), y el olor puede deberse a la descomposición de la materia orgánica de bacterias (Kim et al., 2001) y a veces a la proliferación de cianobacterias (Ferreira et al., 2002) que aprovechan el excedente de nitrógeno disponible, y proliferan mediante asociaciones simbióticas como Anabaena con los helechos del género Azolla. La presencia de de la familia Tubificidae es un indicador típico de los procesos de eutrofización con sedimentos de baja concentración orgánica (Lang, 1997).

Un aspecto importante a destacar, es que en los sitios donde el proceso eutrófico es menos conspicuo, se encontró mayor cobertura de Schoenoplectus californicus ssp. totora, que actúa como un agente de descontaminación natural de las aguas (especialmente el nitrógeno) por sus propiedades fisiológicas (Meagher, 2000), aumentando de esa manera, la capacidad buffer del lago, que está en función a la resilencia del ambiente (Carpenter \& Cottingham, 1997; Petrucio \& Esteves, 2000).
A pesar de la evidente utilidad de estos indicadores, la eficacia de los mismos no es del $100 \%$, ya que no todos los ecosistemas responden de la misma manera a las perturbaciones ambientales y las interacciones de los factores climáticos, biológicos, físicos y químicos muchas veces dan resultados no esperados. Indicadores como la cantidad y diversidad de fitoplancton y la presencia de macrófitas flotantes invasoras son de un alto nivel de confiabilidad, mientras que indicadores como el $\mathrm{pH}$, la presencia / ausencia de ciertos grupos de fauna, la cobertura relativa de macrófitas sumergidas y el olor son indicadores más subjetivos porque dependen de factores externos, a veces difíciles de cuantificar o predecir.

Si bien el proceso eutrófico en el lago Titikaka aun está localizado solamente en áreas próximas a asentamientos humanos importantes, si la contaminación persiste con los niveles actuales de emisión puede derivarse en una situación peligrosa para la salud de los pobladores por el metabolismo de tóxicos y toxinas (Ferreira et al., 2002; Sohrin et al., 1997), como es el caso de las bahías de Puno y Cohana (Northcote, 1991). Cuando el proceso eutrófico es aun poco significativo es relativamente sencillo controlar a las especies invasoras mediante compuestos químicos como el TBT (Zhihui \& Tianyi, 1998) o mediante la construcción de fotobioreactores sencillos (Baumgarten et al., 1999), pero una vez avanzado el proceso eutrófico, la recuperación del 
lago sería un proceso largo, difícil y costoso (Myrbo \& Ito, 2003).

Sería recomendable realizar un estudio más profundo del avance del proceso eutrófico en el lago Titikaka, tomando en cuenta los parámetros indicadores mencionados y realizando mediciones de parámetros fisicoquímicos y de diversidad que permitan construir un modelo de simulación dinámica (Heiskary, 2000) que sería de mucha utilidad para el monitoreo del proceso, y también debería plantearse un modelo social-ambiental para gestión del problema como sugieren Janssen \& Carpenter (1999).

\section{Conclusiones}

Se percibió que los sitios cercanos a asentamientos humanos están más expuestos a la contaminación y a la descarga de contaminantes ricos en nutrientes, y están desarrollando, todavía de manera localizada y temprana, procesos de eutrofización.

El uso de los indicadores planteados se muestra como una herramienta útil para la evaluación rápida de la eutrofización localizada a orillas del Lago Titikaka. Sin embargo, para un estudio integral estos indicadores deberían complementarse con otro tipo de estudios fisicoquímicos y de diversidad más profundos, puesto que los indicadores biológicos a veces son contradictorios y no son $100 \%$ confiables por la influencia de externalidades ambientales.

Es recomendable el proseguir con estudios más profundos y detallados sobre esta problemática, que permitan construir un modelo socio-ambiental adecuado para la gestión de esta problemática.

\section{Agradecimientos}

A Gabriela Mirones por su apoyo y ayuda incondicional. Al equipo de trabajo del proyecto Telmatobius: Dr. E. Richard, G. García, R. Rocha, M. Ferrufino y N. Brun.

\section{Literatura citada}

Achá D. \& Fontúrbel F. 2003. La diversidad de una Comunidad, ¿Está controlada por Top-Down, Bottom-Up o una combinación de estos?. Revista de Biología.Org. 13: 1-16.

Agatz M., Asmus R.M. \& Deventer B. 1999. Structural changes in the benthic diatom community along a eutrophication gradient on a tidal flat. Helgol Mar Res. 53: 92-101.

Barrón C., Marbà N., Duarte C.M., Pedersen M.F., Lindblat C, Kersting K., Moy F. \& Bokn T. 2003. High organic carbon export precludes eutrophication responses in experimental rocky shore communities. Ecosystems. 6: 144-153.

Baumgarten E., Nagel M. \& Tischner R. 1999. Reduction of the nitrogen and carbon content in swine waste with algae and bacteria. Appl Microbiol Biotechnol. 52: 281-284.
Carpenter S. \& Cottingham K. 1997. Resilience and Restoration of Lakes. Conservation Ecology. 1, art 2.

Dolbeth M., Pardal M.A., Lilleblo A.I., Azeiteiro U. \& Marques J.C. 2003. Short- and long-term effects of eutrophication on the secondary production of an intertidal macrobenthic community. Marine Biology. 10 (1007): 1133-1135.

Ferreira A. 2001. Disponibilidade de nitrogênio, alterações nas características químicas do solo e do milho pela aplicação de efluente de esgoto tratado. MSc., Universidade de São Paulo, São Paulo.: 126.

Ferreira F., Góis M.H., Marques C., Rosas A., Kingwell P. \& Simões I. 2002.Ocorrência de cianobactérias em três praias fluviais, localizadas na rede hidrográfica da bacia do Rio TâmegaPraia Fluvial Aurora, itetos e Pontinha. $6^{\circ}$ Congresso da água, Porto.: 2 .

Fontúrbel F., García G., Brun N., Ferrufino M. \& Rocha R. 2003. Propuesta de producción sostenible de Telmatobius culeus (Anura: Leptodactylidae) en el lago Titikaka, mediante la implementación conjunta de un sistema de control de calidad ambiental. En: Fontúrbel, F. \& G. García (eds.), Propuestas Para Un Desarrollo Sostenible: Lago Titikaka Por Estrategas K. Publicaciones Integrales, La Paz.: 19-52.

García P. 2003.Macrófitos acuáticos en los humedales andaluces. Revista Medio Ambiente. Junta de Andalucía, Sevilla.

Heiskary S. 2000. Simplified Lake Eutrophication Modeling: Using the Ecoregionbased model MINLEAP. Minnesota Pollution Control Agency Minnesota Pollution Control Agency, Minnesota.: 19.

Howarth R., Swaney D.P., Butler T.J. \& Marino R. 2000. Climatic control on eutrophication of the Hudson River Estuary. Ecosystems. 3: 210-215.

Janssen M. \& Carpenter S. 1999. Managing the Resilience of Lakes: A Multiagent Modeling Approach. Conservation Ecology. 3, art 15.

Kim B., Park J.H., Hwang G., Jun M.S. \& Choi K. 2001. Eutrophication in reservoirs of South Korea. Limnology. 2: 223-229.

Koivula K. \& Rönkä A. 1998. Habitat deterioration and efficiency of antipredator strategy in a meadow-breeding wader, Temminck's stint (Calidris temminckii). Oecologia. 116: 348-355.

Lang C. 1997. Oligochaetes, organic sedimentation, and trophic state: how to assess the biological recovery of sediments in lakes? Aquat.sci.. 59: 2633.

Macek T., Macková M. \& Kás J. 2000. Exploitation of plants for the removal of organics in environmental remediation. Biotechnology Advances. 18: 23-34.

Manca M., Ramoni C. \& Comoli P. 2000. The decline of Daphnia hyalina galeata in Lago Maggiore: a 
comparison of the population dynamics before and after oligotrophication. Aquat.sci. 62: 142-153.

McCook L.J. 1999. Macroalgae, nutrients and phase shifts on coral reefs: scientific issues and management consequences for the Great Barrier Reef. Coral Reefs. 18: 357-367.

Meagher R. 2000. Phytoremediation of toxic elemental and organic pollutants. Current Opinion in Plant Biology. 3: 153-162.

Myrbo A. \& Ito E. 2003.Eutrophication and remediation in context: High-resolution study of the past 200 years in the sedimentary record of Lake McCarrons (Roseville, Minnesota). USGSWRRI 104B National Grants Competition and the Center for Agricultural Impacts on Water Quality, Minnesota: 5.

Northcote T. 1991. Eutrofización y problemas de polución. En: Dejoux, C. \& A. Iltis (eds.), El Lago Titicaca: Síntesis Del Conocimiento Limnológico Actual. Hisbol - ORSTOM, La Paz.: 563-572.

Pérez E. 1998. Dieta y ciclo gametogénico anual de Telmatobius culeus (Anura: Leptodactylidae) en el lago Titicaca (Huiñaimarca). Licenciatura, Universidad Mayor de San Andrés, La Paz. : 140.

Pérez E. 2002.Evaluación de la población de la rana gigante en Bolivia. Subcontrato 21.22 del Proyecto de Conservación de la Biodiversidad en la Cuenca del sistema TDPS, ALT-IE-FUNDECO, La Paz.: 114.
Petrucio M. \& Esteves F.A. 2000. Uptake rates of nitrogen and phosphorus in the water by Eichhornia crassipes and Salvinia auriculata. Rev. Brasil. Biol. 60: 229-236.

RAMSAR. 1999.Ramsar COP7 DOC. 24: Especies invasoras y humedales. RAMSAR COP, San José. : 9.

Rodríguez J.A. 2002.¿Cuál es la flora característica de un proceso eutrófico? Sierra de Baza. Vol. 2003.

Schroll H. 2002. Indicators of the Long-term Eutrophication of a Danish lake (Karlsø), and Water Pollution Management. The Journal of Transdisciplinary Environmental Studies. 1: 1-10.

Sohrin Y., Matsui M., Kawashima M., Hojo M. \& Hasegawa H. 1997. Arsenic Biogeochemistry Affected by Eutrophication in Lake Biwa, Japan. ICR Annual Report. 4: 14-15.

Weisner S.E.B., Strand J.A. \& Sandsten H. 1997. Mechanisms regulating abundance of submerged vegetation in shallow eutrophic lakes. Oecologia. 109: 592-599.

Weithoff G., Lorke A. \& Walz N. 2000. Effects of water-column mixing on bacteria, phytoplankton, and rotifers under different levels of herbivory in a shallow eutrophic lake. Oecologia. 125: 91-100.

Zhihui S. \& Tianyi C. 1998. Toxicity of Tributyltin to Lemna minor L. and Azolla filiculoides Lamk. Bull. Environ. Contam. Toxicol. 60: 318-322. 